\title{
Neutrino Absorption Tomography of the Earth's Interior using Isotropic Ultra-high Energy Flux
}

\author{
Pankaj Jain $^{a}$, John P. Ralston ${ }^{b}$ and George M. Frichter ${ }^{c}$ \\ ${ }^{a}$ Physics Department, I.I.T., Kanpur, India 208016 \\ ${ }^{b}$ Department of Physics and Astronomy \\ University of Kansas, Lawrence, KS 66045-2151, USA \\ ${ }^{c}$ Department of Physics, Florida State University, Talahassee, FL 32306-3016 USA,
}

(August 24, 2018)

\begin{abstract}
We study the feasibility of using an isotropic flux of cosmic neutrinos in the energy range of 10 to $10000 \mathrm{TeV}$ to study the interior structure of Earth. The angular distribution of events in a $\sim \mathrm{km}^{3}$-scale neutrino telescope can be inverted to yield information on the Earth's mass distribution that is independent of other methods. The energy spectrum of the neutrino primaries is also determined from consistency with the angular distribution. It is possible to make a model independent determination of the density profile of Earth's interior, separate from the absolute normalization of the incident cosmic neutrinos.
\end{abstract}

The nature of the Earth's interior has traditionally been deduced by indirect physical methods. An early, noteworthy result was Cavendish's 1793 deduction that the Earth must have a dense core, obtained by "weighing the Earth" gravitationally. Current measurements are based largely on seismic wave propagation, which is rather indirect and has substantial intrinsic uncertainties [1]. Adding extra information fails to remove ambiguitites, including studies ranging from the vibrational modes of the Earth as an elastic body, to temperature constraints [2], to the detailed composition of the core [2]. Controversies currently exist: for example, seismic data has indicated that there may be an unsymmetrical differentially rotating element in the core [3], with contenders to explain this including a very large single crystal. Independent measurements of the density profile would be of considerable value. Here we discuss a novel way to take a rather direct 'snapshot' of the nucleon density in the Earth's interior, by considering tomography with ultra-high energy neutrinos of cosmic origin. The principle of neutrino tomography is essentially the same as X-ray tomography, except for substituting penetrating neutrinos to serve in place of X-rays. By measuring neutrino absorption along different paths through a solid body, one can deduce the nucleon density in the interior of the object. The results would be utterly independent of the geophysical model, and directly measure the nucleon density.

The interaction strength of neutrinos with other fundamental particles increases strongly with energy, and has been well measured at several high energy accelerators [4]. The energy range of these direct measurements runs from below $10^{8} \mathrm{eV}$ up to almost $10^{14} \mathrm{eV}$, the latter being achieved recently at the accelerator HERA in Hamburg. For almost all of the ultra-high energy (UHE) region of incident energy above $10^{13} \mathrm{eV}$, the cross section has not been measured directly. However, $\sigma$ can be calculated by exploiting relations in the Standard Model between electron-initiated reactions which have been measured, the neutrino initiated reactions desired, and evolution of active quark and anti-quark pairs with energy [5.6]. The uncertainty due to theory in these calculations is small, leading to a fundamental interaction which is sufficiently well known for the purposes of tomography.

The flux of UHE neutrinos from cosmic sources cannot yet be considered established, and several pilot experiments in the $\mathrm{TeV}\left(1 \mathrm{TeV}=10^{12} \mathrm{eV}\right)$ energy range are underway to measure it. The BAIKAL experiment operates in lake water; the AMANDA project detects neutrinos interacting in the Antarctic ice cap. A third scheme called RICE exists in the pilot stage, and uses a novel radio detection strategy which is the most effective method above 100 $\mathrm{TeV}$. The optimal energy range for neutrino tomography is roughly 10-1000 TeV, a region where these existing pilot projects have some overlap. However, current detectors are small and would give a marginal or insufficient event rate for Earth tomography. With better resources, employing a few hundred optimally tuned detectors, our calculations indicate that one should be able to say something useful about the Earth's interior. However our primary focus is a future detector on a much larger scale, with a detection volume of order $1 \mathrm{~km}^{3}$ (KM3). With fluxes of the order of current astrophysical estimates, a KM3 detector should be able to perform definitively.

Our approach differs from previous studies of neutrino absorption tomography. The older studies concentrated on exploiting point neutrino sources assumed to have a steady time dependence [7 14]. Reconstruction of the density profile is done by observing periodic occultation of the sources due to the Earth's rotation. This method relies on the rate obtained from limited point sources, and is also subject to errors if the energy dependence of the primary spectrum is poorly determined. The geometry will not work for a detector located at the South Pole. Kuo et al. 14] investigated Earth tomography in the context of the DUMAND II array, concluding that a time scale of 'from 
years to decades' was needed to obtain sufficient data with this method. These results are important, but we offer a complementary and more promising scheme. We consider neutrinos coming from unresolved active galactic nuclei, gamma ray bursts, secondary emissions from cosmic rays whose directions are scrambled by cosmic magnetic fields, and other possible galactic or cosmological sources of ultra high energy neutrinos. Integrating over the Universe, this diffuse flux should be nearly isotropic, with a sizable component in the optimal energy region. Such a flux has several advantages. For example, one overcomes the serious problem of binning events by arrival times to incorporate the effects of the Earth's rotation. There is far less ambiguity due to any possible time dependent fluctuations in the flux of an energetic point source. Most attractively, the entire Earth density profile can be obtained unambiguously, by a simple inversion of a well-measured observable in the data, namely the angular distribution. The overall normalization of the flux is not needed, as we arrange the calculation so that it drops out of the determination of the density profile.

The energy dependence of the primary neutrino flux is also determined by the procedure. This is unexpected and rather miraculous, but it occurs because the interaction cross section and detector efficiencies are energy dependent. Given initial data on the angular distribution, and supposing poorly determined initial data, or guesses, on the energy spectrum (always a problem in cosmic ray physics), our procedure iterates the energy spectrum to obtain consistency with the angular distribution and density. Put another way, a faulty energy spectrum would be inconsistent, and by iteration the angular distribution and measured energy flux after attenuation determine the energy spectrum. This is quite interesting and may serve as a good method to measure the incident energy spectrum. If one assumes that the density profile of the Earth is already well known, then the angular distribution strongly overdetermines the problem, and one might even deduce the energy dependence of the cross section, contributing a powerful check on fundamental physics.

To illustrate these remarks, consider the angular distribution of neutrinos passing through the Earth (Fig. 1). With an isotropic primary flux, the angular dependence comes from differing amounts of matter traversed en route to the detector. The effect is expressed by an evolution equation for the flux, $\Phi$, as a function of distance $z$ traversed:

$$
\frac{d \ln \Phi(E, z)}{d z}=-n(z) \sigma_{\mathrm{eff}}(E) .
$$

Here $\sigma_{\text {eff }}$ is a known 'effective' cross section which incorporates both charged current cross sections, neutral current cross sections, and neutral current regeneration [15.16] for neutrinos of energy $E$. We measure the polar angle $\theta$ with respect to the nadir (Fig. 1). We assume spherical symmetry, so that the density $n=n(r)$ is a positive definite function of distance $r$ from the Earth's center. Let us assume momentarily that the measurement is dominated by a sufficiently narrow range of neutrino energies, so that the variation of (1) with energy can be neglected. By taking the logarithm of the flux, the overall flux normalization is an additive constant that drops out of the angular distribution.

Solving (11) for the surviving neutrino flux that can be measured at a detector site located near the Earth's surface, one obtains,

$$
\Phi_{\text {surv }}(E, \theta)=\Phi_{\nu}(E) e^{-\sigma_{\text {eff }}(E) R n(R) f(\theta)},
$$

where $\Phi_{\nu}$ is the incident neutrino flux, $R$ is the Earth's radius and the function $f(\theta)$ is proportional to the integrated nucleon density along the chord $0<z<2 R \cos (\theta)$. It is convenient to measure $r$ in units of the Earth's radius $R$. Then,

$$
f(\theta)=\frac{1}{n(R)} \int_{\sin ^{2}(\theta)}^{1} \frac{n(r) d\left(r^{2}\right)}{\sqrt{r^{2}-\sin ^{2}(\theta)}} .
$$

Given data for the angular dependence over the region $0<\theta<\frac{\pi}{2}$, this particular transform can be inverted; the result is:

$$
n(r)=-\frac{n(R)}{\pi} \int_{\sin ^{-1}(r)}^{\frac{\pi}{2}} \frac{d f(\theta)}{d \theta} \frac{d \theta}{\sqrt{\sin ^{2}(\theta)-r^{2}}} .
$$

This analytic result shows that the angular distribution is sufficient to give the density profile. The result is simpler than might be expected, because the particular spherical geometry of the problem has been exploited. Deviations from spherical symmetry are of interest, so that relaxing our assumptions can be contemplated, but our goal here is to prove the practicality of the simplest scheme when confronting realistic difficulties. The primary difficulty appears to be statistical fluctuations from small expected data sets, but we will find that these appear to be under control on the scale of KM3.

We now turn to the question of energy dependence that was sidestepped above. The simple procedure above can be applied within a small energy bin. One might then imagine requiring that in each angular bin, we also bin the 
data in energy. With limited statistics and limited energy resolution of the detector, we find that such a method is unlikely to be practical. Yet integrating over energy does not commute with the angular inversion, so a priori the energy integrated angular distrbution does not appear to be adequate. To get around these problems, we created an alternative procedure in which the Earth's density profile and the incident flux is iteratively improved.

We start by assuming a trial function for the density profile, which will yield our initial guess for the attenuation factor $f(\theta)$. This can be used, along with data on the energy dependence of observed flux integrated over nadir angle, to obtain our first guess for the incident energy spectrum. The attenuation function $f(\theta)$ can then be further improved by using the calculated value of the incident flux. Again this is compared with the angular distribution of the observed flux integrated over energy. At no stage is it necessary to have the joint distribution in energy and in angle. This procedure is repeated till convergence is obtained for both the incident flux and $f(\theta)$. The same procedure has then converged to the Earth's density profile. In order to obtain convergence and a unique solution, it is necessary to fix one boundary condition, which is taken to be the value of the density of the Earth near the surface. Since the surface density is known with reasonable accuracy, the boundary condition should not introduce any bias.

In fact the result is overdetermined, because two moments of the density are already known: These are the total mass of the Earth (just as Cavendish used), and the Earth's moment of inertia. But the entire procedure can be carried out without making use of these moments, so in practice the density is over-determined. This is important, because one can expect only crude measurement of the energy distribution from a realistic cosmic ray detector.

We used standard Monte Carlo methods to study the feasibility of the iterated inversion technique. The simulations used the Preliminary Reference Earth Model (PREM) 17] for the Earth's density profile. The range of neutrino energies was restricted to lie between $10-10^{4} \mathrm{TeV}$. Our numerical results show that the optimal lower limit in energy is between 10 and $50 \mathrm{TeV}$, since below this value the Earth is essentially transparent. The optimal upper limit is between $10^{3}$ and $10^{4} \mathrm{TeV}$, beyond which the number of events are expected to be too small to be of much use for tomography. We employed a generic form of the diffuse AGN neutrino flux, $\Phi_{\nu}(E)=\Phi_{o} E^{-2}$ for $10 \mathrm{TeV}<E<10^{4} \mathrm{TeV}$. This form is within the range of current theoretical predictions. For example, in the energy range of interest, the AGN model of Stecker, Done, Salamon and Sommers (SDSS) 18 gives a flux $\sim E^{-1}$. A model due to Szabo and Protheroe (SP) [19], while not now thought to be correctly normalized, has the neutrino spectrum falling like $\sim E^{-2}$. We take an agnostic position on the flux, and address uncertainties by simply renormalizing results at the end. We simulated data for a generic UHE neutrino telescope, for the purposes of study defined in two ways: in one extreme for simplicity, we took detector response independent of neutrino energy and angle of incidence. The other extreme is the case of detector with the energy and angular response calculated for a radio array [16]. The radio method has an response strongly increasing with energy, making the flat response to the higher-energy part of the spectrum a more conservative method. Meanwhile we do not have sufficient information on the angular response of optical detection. Since we believe that a realistic detection scheme would combine the strengths of both optical and radio detection, the two cases should give a reasonable range of results without excluding either or getting bogged down in details: in fact, the results were so similar that we simply report the simpler (isotropic and flat) response. Of course, detector response for a particular experimental situation, as well as realistic energy resolution and pointing accuracy, can always be incorporated.

Our simulation generated $N$ events distributed in nadir angle, $0^{\circ}<\theta<90^{\circ}$, and energy, $10 \mathrm{TeV}<E_{\nu}<10^{4} \mathrm{TeV}$, according to Eqn. (2). We normalized our calculation so the total number of events observed per antenna between $100 \mathrm{TeV}$ and $10^{4} \mathrm{TeV}$ is about 100 per year. This is about one fourth of the event rate calculated in [16], which takes into account subsequent changes in estimates of the incident flux. While we thus normalize our rates to radio detection, any combination of methods can be rescaled in an obvious way.

The Monte Carlo data were divided into 20 energy $E$ bins, with widths increasing like $E^{2}$. The data was also divided into 10 angular bins chosen to be equally spaced in radius from the center of the Earth. These bins were chosen to obtain the density at roughly uniform intervals. We made no attempt to discover an optimal binning procedure. However the solid angle subtended by the central bin, namely the one containing the center of Earth, is very small, and hence it puts severe requirements on the total number of events needed to say something useful about the density in this region. This also happens to be one of the most interesting regions for geophysics. Depending on the total number of events and optimization scheme, one may wish to adjust this bin to get a better measurement.

We found that for a wide range of trial density profiles convergence of the iterative procedure was obtained within about 5 iterations. We plot in Fig. 2 an example of successive approximations to the attenuation function $f(\theta)$, with the solid line showing the final result. Fig. 3 shows the successive approximations for the incident flux in energy. The plots show rapid convergence occurs with the scheme chosen. The figures show explicitly that a considerable error in the incident energy spectrum can be tolerated, with the final energy spectrum converging to the actual spectrum.

The final extracted density profile, along with the PREM density profile, is shown in Fig. 4. The step between the core and lower mantle is very well resolved, while the inner core is not. Since the density converged to proper value, then all of its moments also converged, showing that the total mass and moment of inertia were consistent, or: Neutrinos can "weigh the Earth". In practice the known mass density moments provide an excellent handle on the 
overall consistency of the final result. Statistical error bars on the derived density are acceptable (Fig. 4), although one would probably want to optimize the central core region further. The results were obtained by assuming that a detector with 1000 antennas is deployed for two years. The number is ambitious but within the scope of planning for future arrays. Because the errors are statistical, the same result would be obtained for an incident flux normalized 5 times lower in 10 years running. Put yet another way, even a modest array of 200 antennas might say something useful in the time scale of 10 years. In Fig. 5 we also show the final result assuming the optimistic flux estimates, but only 100 antennas for a running time of 2 years. In this case we divide the earth's radius into only five bins instead of ten, in order to get a reasonable number of events in the central bin. The step between the core and lower mantle remains well resolved.

We hasten to add that there can be many uncertainties in realistic experimental design, which only further study can address. During a period of a few years to a decade, a $K M 3$ neutrino telescope will be fulfilling a primary mission as a fundamentally new kind of instrument for observing the cosmos. We believe that with the same kind of detector, neutrino tomography could also provide important and independent information about the Earth's interior.

Acknowledgements: We thank Geoff Abers, John Doveton, Doug McKay and R. P. Singh for useful comments. Supported by DOE grant number DE-FGO2-98ER41079, the KU General Research Fund, NSF-K*STAR Program under the Kansas Institute for Theoretical and Computational Science and DAE grant number DAE/PHY/96152.

[1] See current text on seismology, e.g. Mordern Global Seismology, by T. Lay and T. C. Wallace ( Academic Press, 1995); Quantitative Seismology: Theory and Methods, Vols 1 and 2, by K. Aki and P. G. Richards, (W. H. Freeman, 1980).

[2] R. Jeanloz, Ann. Rev. Earth Plan. Sci. 18, 357 (1990); R. Jeanloz and S. Morris, ibid 14, 377 (1986).

[3] X. D. Song and P. D. Richards, Nature 382, 221, (1996); W. J. Su, A. M. Dziewonski, and R. Jeanloz, Science 274, 1883 (1996); X. D. Song, Rev. Geophys. 35, 297 (1997).

[4] Particle Data Group, European Physical Journal C, 3 (1-4) (1998).

[5] G. M. Frichter, J. P. Ralston, and D. W. McKay, Phys. Rev. Lett. 74, 1508-1511 (1995).

[6] R. Gandhi, C. Quigg, M. H. Reno, I. Sarcevic, Phys. Rev. D 58 (1998) 093009.

[7] L. V. Volkova and G. T. Zatsepin, Bull. Phys. Ser. 38, 151-154 (1974).

[8] A. De. Rujula, S. L. Glashow, R. R. Wilson and G. Charpak, Phys. Rep. 99, 341-396 (1983).

[9] T. L. Wilson, Nature 309, 38-42 (1984).

[10] G. A. Askar'yan, Usp. Fiz. Nauk 144, 523-530 (1984); Sov. Phys. Usp. 27, 896-990 (1984).

[11] A.B. Borisov, B.A. Dolgoshein, and A.N. Kalinovskii, Yad. Fiz. 44, 681-689 (1986); Sov. J. Nucl. Phys. 44,442 (1987).

[12] A. Nicolaidis, M. Jannane, and A. Tarantola, J. of Geophys. Res. 96, 21811-17 (1991).

[13] H. J. Crawford, R. Jeanloz, B. Romanowicz, and the DUMAND collaboration, Proc. of the XXIV International Cosmic Ray Conference, (University of Rome), 804 (1995).

[14] C. Kuo, H. J. Crawford, R. Jeanloz, B. Romanowicz, G. Shapiro and M. L. Stevenson, Earth and Planetary Science Letters 133, 95 (1995).

[15] V. S. Berezinsky, A. Z. Gazizov, A. Z. Rozental and G. T. Zatsepin, Sov. J. Nucl. Phys. 43, 406 (1986).

[16] G. M. Frichter, J. P. Ralston, and D. W. McKay, Phys. Rev. D53 1684 (1996).

[17] A. M. Dziewonski and D. L. Anderson, Phys. Earth Planet. Inter., 25, 297-365 (1981).

[18] F. W. Stecker, C. Done, M. H. Salamon and P. Sommers, Phys. Rev. Lett. 66, 2697; E (1992): ibid 69, 2738.

[19] A. P. Szabo and R. J. Protheroe, Astropart. Phys. 2, 375 (1994); R. J. Protheroe and A. P. Szabo, Phys. Rev. Lett. 69, 2285 (1992). 


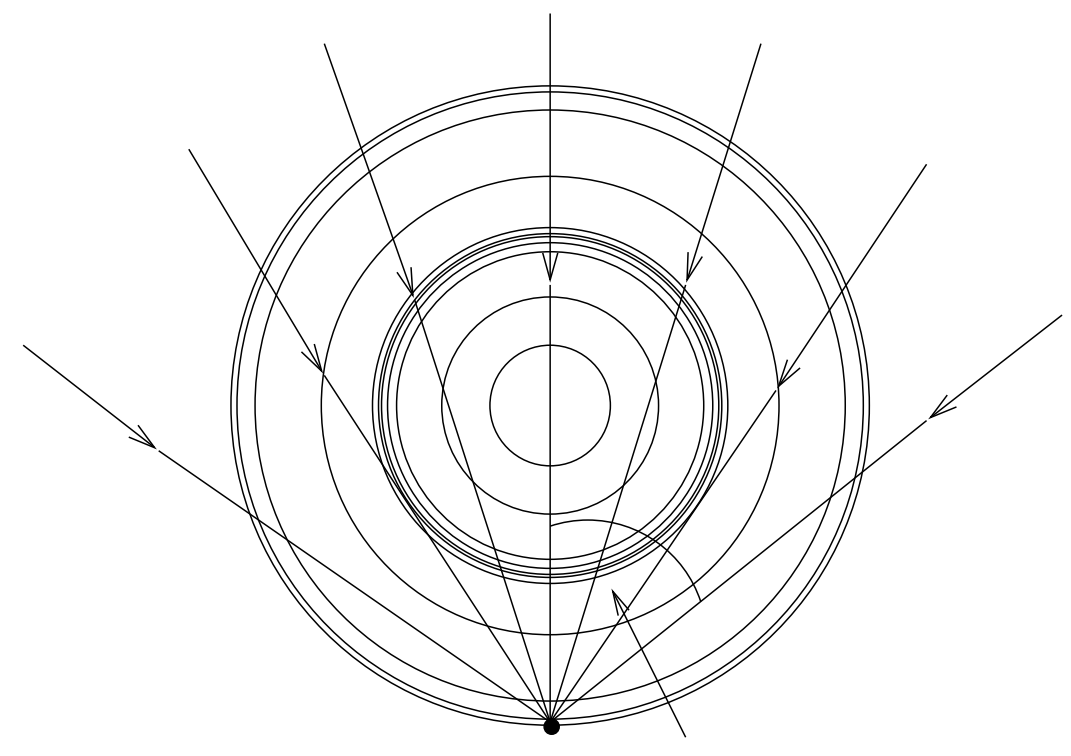

$\Theta$

FIG. 1. Earth density contours, and paths traced by netrinos at different angles of incidence to the detector. 


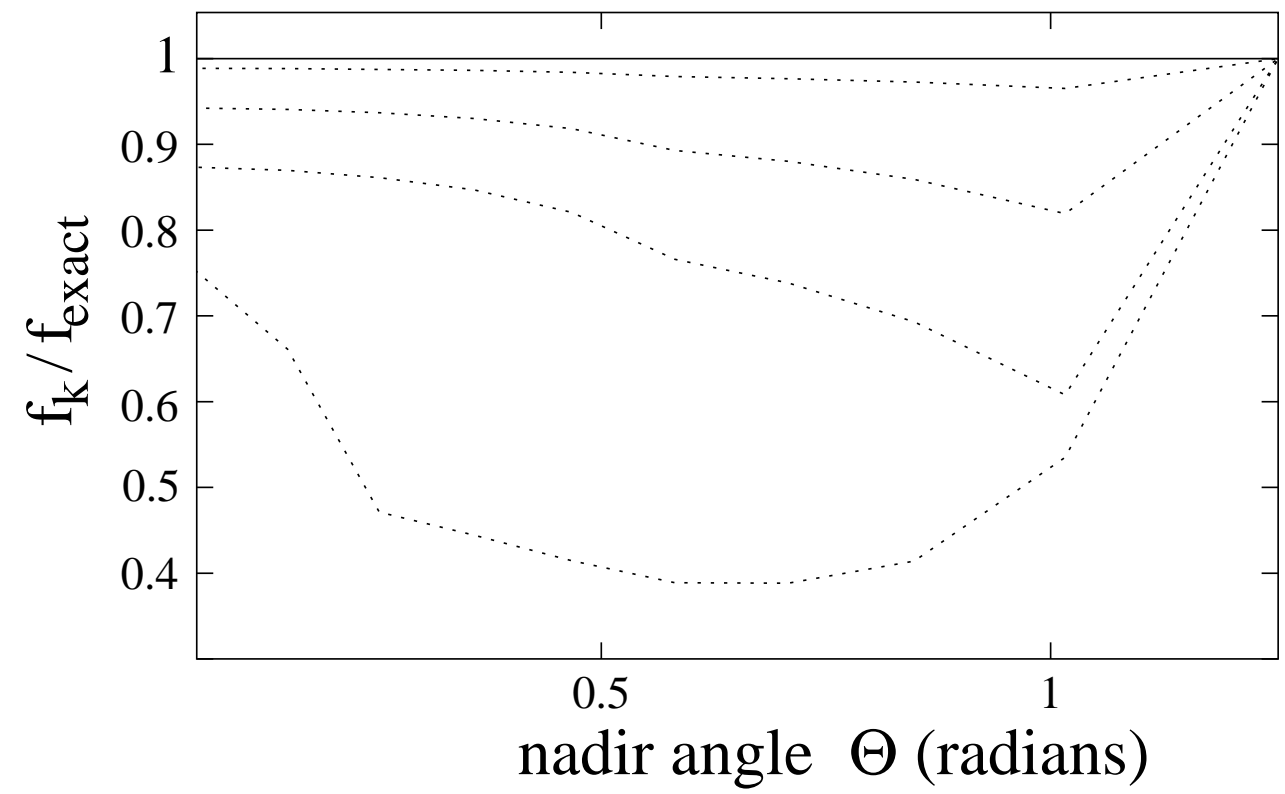

FIG. 2. Successive approximations to the attenuation function $f(\theta)$ calculated iteratively, where $\theta$ is the nadir angle. The solid line shows the final result

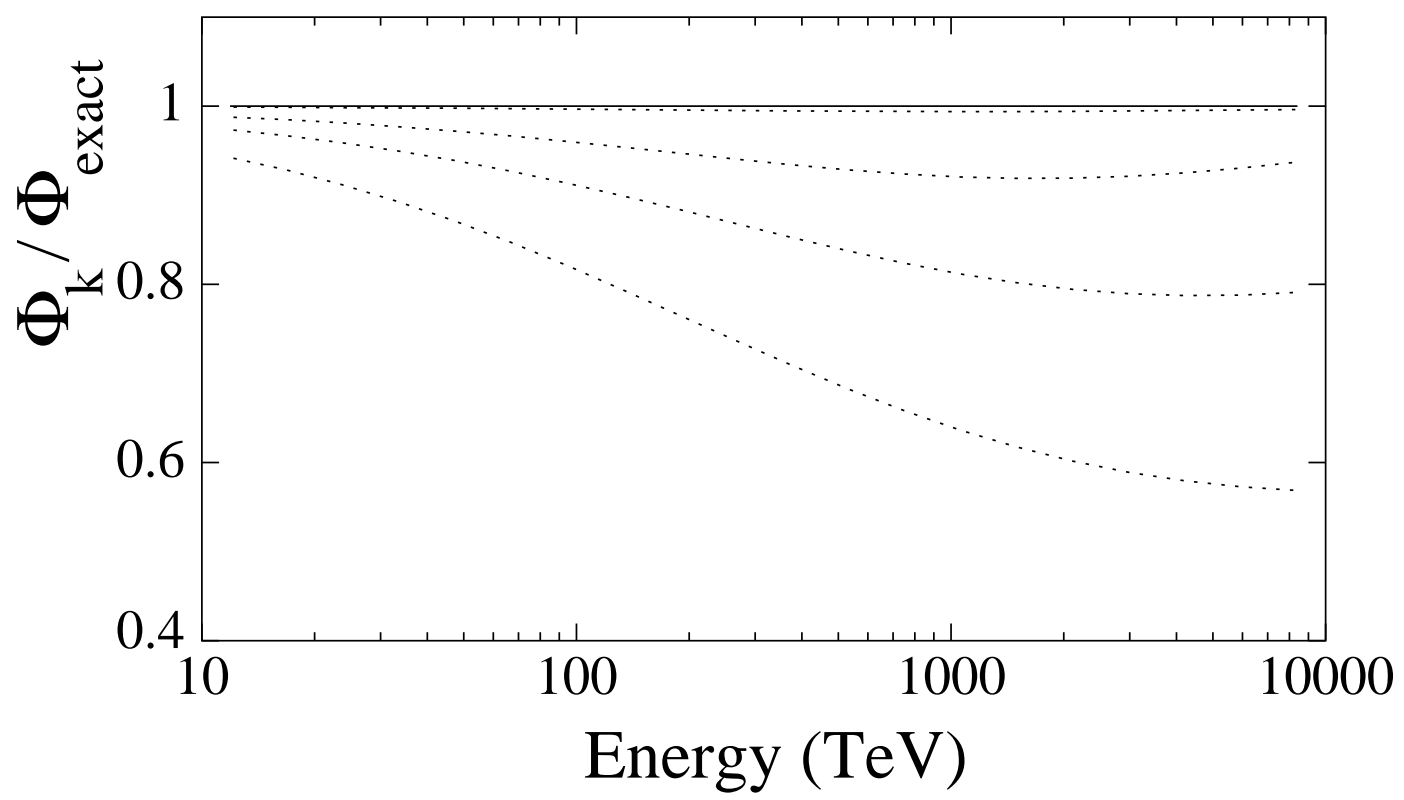

FIG. 3. Successive approximations to the extracted value of the incident flux $\phi(E)$, calculated iteratively. The solid line shows the final result. 


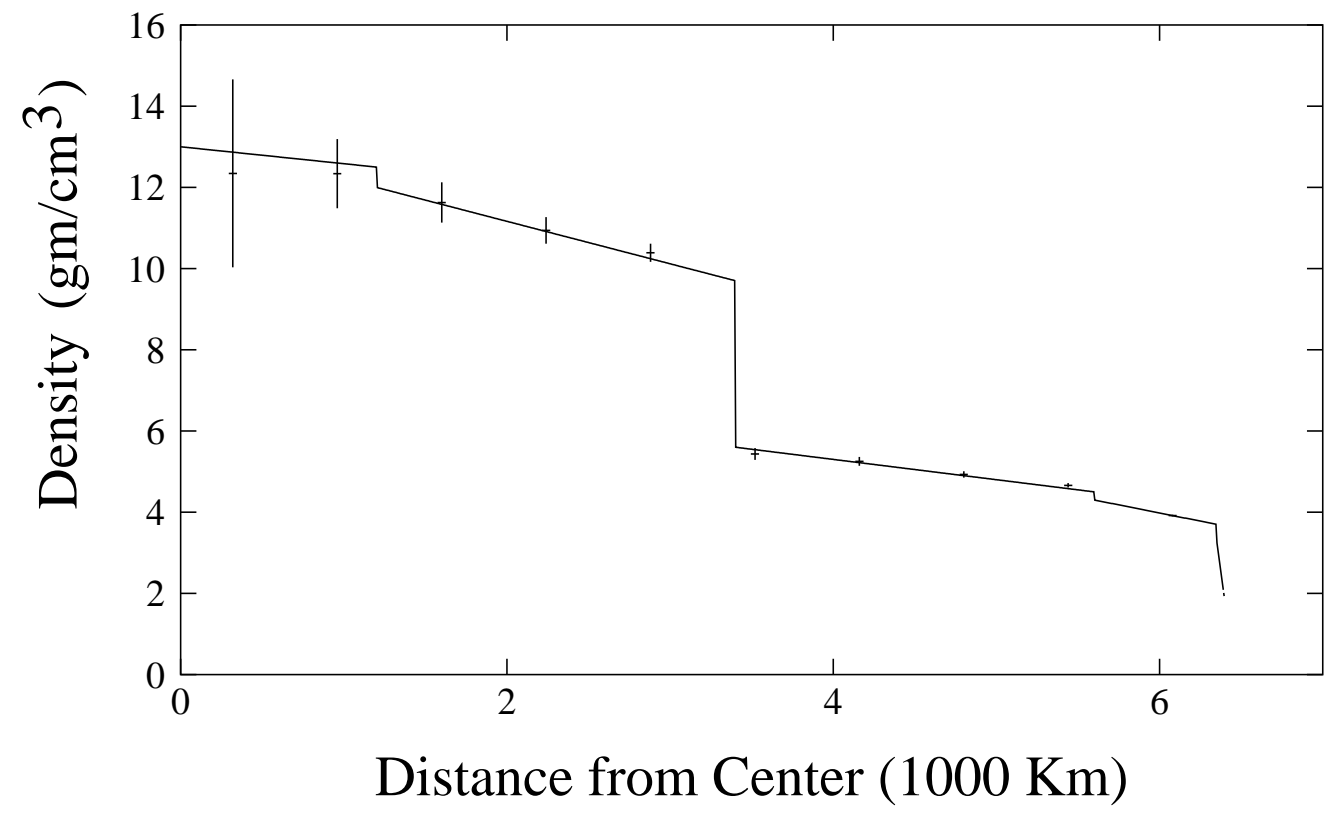

FIG. 4. The extracted density using ten radial bins along with the statistical errors. The PREM model for the Earth's density, solid curve, was used to generate data. The step between the core and lower mantle is very well resolved, while the difference between the inner and outer cores is not.

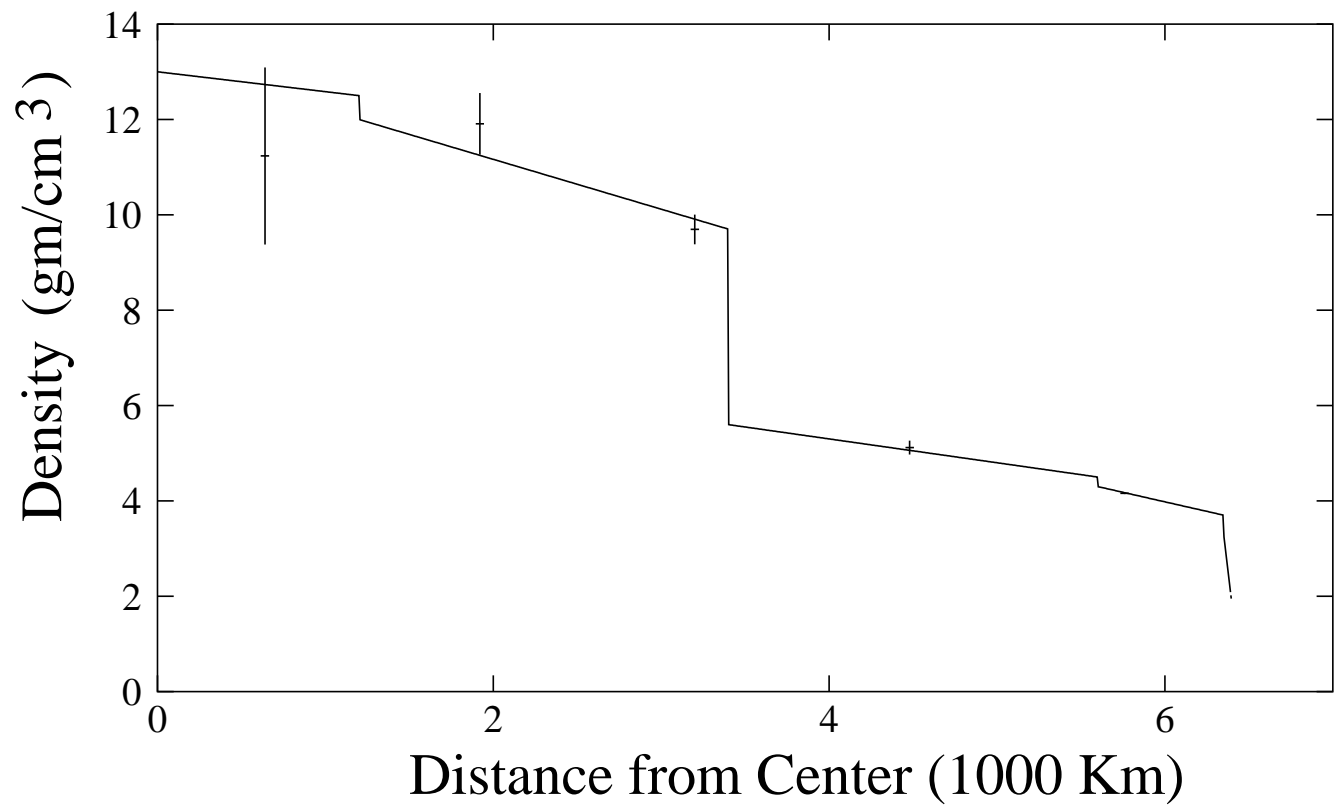

FIG. 5. The extracted density using five radial bins along with the statistical errors, assuming one tenth the event rate compared to Fig. 4. The step between the core and lower mantle remains well resolved. 